\title{
Treating to hypertension targets
}

\section{A McKerracher}

Heart 2004;90(Suppl IV):iv33-iv35. doi: 10.1136/hrt.2004.037614

Control of blood pressure levels in hypertensive patients often fails to meet the targets suggested by the British Hypertension Society (BHS) guidelines. The optimal treatment target for non-diabetic patients is $\leqslant 140$ / $85 \mathrm{~mm} \mathrm{Hg}$. Data from the health survey for England show that in 1998 only $8 \%$ of men and $11 \%$ of women had blood pressure controlled to $<140 / 90 \mathrm{~mm} \mathrm{Hg}$. The AngloScandinavian cardiac outcomes trial (ASCOT) data show that $87 \%$ of non-diabetic patients in the St Mary's Centre are currently treated to below $140 / 90 \mathrm{~mm} \mathrm{Hg}$. These differences can be explained by strict protocol adherence, a focus on cardiovascular disease, longer consultation times and punctual appointments, reinforcement of nonpharmacological advice, and blood pressure readings taken in line with BHS guidelines. Some of these points might be translated into the more challenging setting of primary care.
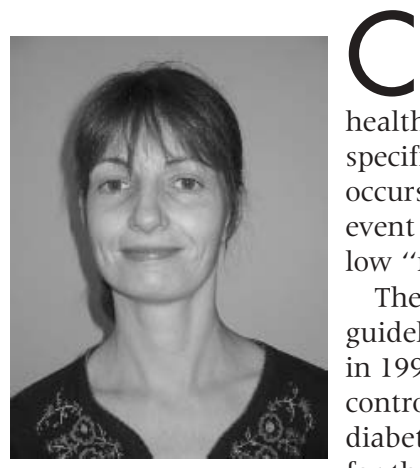
ontrolling blood pressure is of paramount importance in the prevention of cardiovascular disease and remains a challenge for health care professionals. Although there is no specific level at which a cardiovascular event occurs, it is known that there is less risk of an event occurring if blood pressure is lowered to a low "normal" level.

The British Hypertension Society (BHS) issued guidelines for the management of hypertension in 1999. ${ }^{1}$ These guidelines set target levels for the control of blood pressure: for those without diabetes, the target was $\leqslant 140 / 85 \mathrm{~mm} \mathrm{Hg}$ and for those with diabetes it was $\leqslant 140 / 80 \mathrm{~mm} \mathrm{Hg}$. In addition, an audit standard-the minimum acceptable level of control-was set at $\leqslant 150$ / $90 \mathrm{~mm} \mathrm{Hg}$ for those without diabetes and $\leqslant 140 / 85 \mathrm{~mm} \mathrm{Hg}$ for those with diabetes.

The cross sectional health survey for England looked at the management of hypertension in adults in 1994 and again in 1998. ${ }^{2}$ The results showed that there is a high prevalence of hypertension which is untreated according to current BHS targets. Table 1 shows that awareness, treatment, and control rates in 1998 had increased compared with 1994. Nevertheless, in 1998 only $8 \%$ of men and $11 \%$ of women had blood pressure controlled to $\leqslant 140 / 90 \mathrm{~mm} \mathrm{Hg}$.

Correspondence to: A McKerracher, St Mary's Hospital, Praed Street, London W2 INY, UK; a.mckerracher@ imperial.ac.uk

\section{THE ASCOT STUDY}

The Anglo-Scandinavian cardiac outcomes trial (ASCOT) is an ongoing multicentre prospective randomised trial designed to compare two blocker with and without an angiotensin converting enzyme inhibitor and $\beta$ blocker with or without a diuretic - in the primary prevention of coronary heart disease in more than 18000 hypertensive patients at risk of cardiovascular events. ${ }^{3}$ An $\alpha$ blocker was given as third line treatment in both arms. In addition, a proportion of patients were randomised to receive atorvastatin $10 \mathrm{mg}$ or placebo.

Patients were recruited from general practice and more than $85 \%$ were already receiving treatment for hypertension. The mean blood pressure at entry to the study was 164/ $95 \mathrm{~mm} \mathrm{Hg}$. Figure 1 shows that the mean blood pressure fell dramatically over the first year of the study. The lipid arm of the study was closed at three years, at which point the mean blood pressure was 138/80 mm Hg for both groups. The blood pressure lowering arm of ASCOT is ongoing.

At the St Mary's Hospital ASCOT centre we have 872 patients. Among the non-diabetic patients, $87 \%$ have their blood pressures controlled to below 140/90 mm Hg. Among our diabetic patients, $60 \%$ have their blood pressure controlled under 130/80 mm Hg, a slightly lower level than the BHS guideline target of 140/ $80 \mathrm{~mm} \mathrm{Hg}$.

\section{HOW TARGET BLOOD PRESSURES WERE OBTAINED}

Our success in the ASCOT clinic at reaching hypertension targets with the majority of patients can be put down to several factors. Firstly, the clinic has a primary focus on cardiovascular disease: this is our specialty and we do not treat or investigate anything that is not associated with hypertension. Therefore we can afford to concentrate our expertise in reducing blood pressure levels.

All staff at the clinic adhere to a strict protocol of increasing medication. If blood pressure targets are not reached in any individual subject, that person's medication is increased. Even if the average reading is $141 \mathrm{~mm} \mathrm{Hg}$ systolic we will increase medication, providing previous readings have not been low. Temperature, illness, stress, weather, time of day, and so on are not reasons for failing to increase medication. Occasionally we may suggest that a patient returns in a couple of weeks for a re-check when there are extenuating circumstances.

At each visit, clinic staff will check whether or not the subject has taken their medication in the last 24 hours and if tablets have been missed in the past few weeks. It is important for staff to be certain of this before making any changes to antihypertensive regimens-calcium channel
Abbreviations: ASCOT, Anglo-Scandinavian cardiac outcomes trial; BHS, British Hypertension Society 
Table 1 Trends in awareness, treatment and control of hypertension between 1994 and $1998^{2}$

\begin{tabular}{|c|c|c|c|c|}
\hline & \multicolumn{2}{|l|}{ Men } & \multicolumn{2}{|c|}{ Women } \\
\hline & 1994 & 1998 & 1994 & 1998 \\
\hline \multicolumn{5}{|l|}{ Old definition ( $\leqslant 160 / 95 \mathrm{~mm} \mathrm{Hg}$ ) } \\
\hline Percentage aware & 60 & 68 & 66 & 74 \\
\hline Percentage treated & 45 & 54 & 54 & 64 \\
\hline $\begin{array}{l}\text { Percentage controlled } \\
\text { New definition }(\leqslant 140 / 90 \mathrm{~mm} \mathrm{Hg})\end{array}$ & 27 & 37 & 32 & 40 \\
\hline Percentage aware & 33 & 40 & 46 & 52 \\
\hline Percentage treated & 20 & 26 & 32 & 38 \\
\hline Percentage controlled & 5 & 8 & 7 & 11 \\
\hline
\end{tabular}
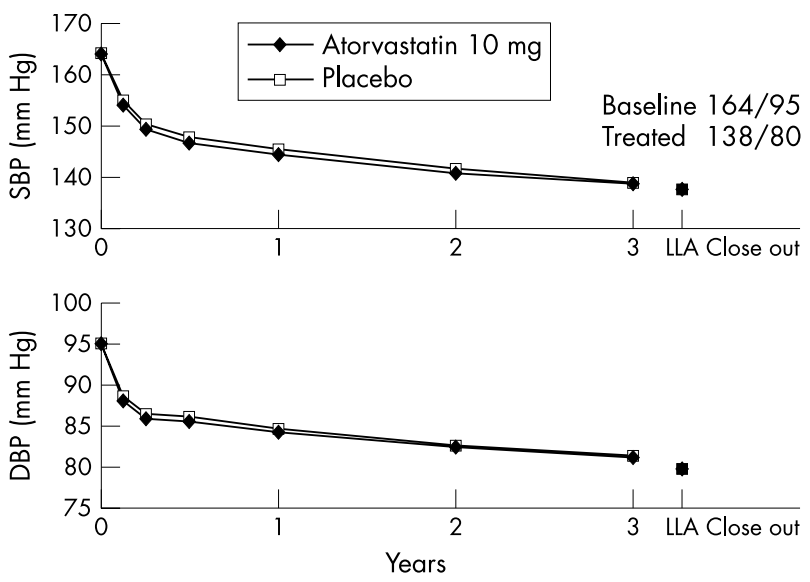

Figure 1 Mean blood pressure changes over the first three years in the ASCOT study (further details available at www. ascotstudy.org).

treatment. Patients are asked to bring all study drugs and other prescribed medications they are currently taking to each visit. The nurse will then ask the patient to explain what they are actually taking. It is surprising how often patients do not take what is prescribed or are taking the wrong tablets. Side effects are a common cause of non-compliance with medication and patients are asked at each visit if they are experiencing any problems with their medication.

More than $80 \%$ of patients attending the clinic are taking more than one antihypertensive medication, and $40 \%$ are taking more than three different drugs. Combining different classes of drugs has been shown to be the most effective strategy for reducing blood pressure. Too many patients are still taking only one drug and are poorly controlled. The clinic follows BHS recommendations into the most effective combinations to use with various groups of patients (fig 2). ${ }^{4}$

The usual ASCOT clinic appointment is 30 minutes long, compared with the 7.5 minutes for a general practitioner appointment. This additional time gives us the opportunity to assess the patient's blood pressure, weight, and general condition, and to undertake blood and urine sampling, review their past blood results and provide non-pharmacological advice as well as tablet dispensing. This is all done in a relaxed environment with the offer of tea or coffee at the end of the appointment if patients have been fasting. There are rarely more than three people in the waiting room at any one time.

Appointments are punctual. This is important as we recognise that all patients are volunteers. Patients also become frustrated if they are kept waiting, reflected in a rise in blood pressure.

The electronic blood pressure devices that we use to measure blood pressure have been validated by the BHS, and are recalibrated yearly to ensure they are reading accurately. The blood pressure taking skills of each nurse are assessed when joining the clinic and familiarity with all blood pressure devices used is essential.

Blood pressure measurements are always taken in accordance with BHS guidelines: patients' arms are measured at each visit to ascertain cuff size, and patients sit for five minutes before their blood pressure is taken. Three readings are taken at least one minute apart and there is no conversation during this time. Sometimes the nurse may leave the room if we feel our presence is causing a rise in pressure.

Non-pharmacological advice is given at each visit, even for patients who have been coming to the clinic for several years. We tend to focus on one area of lifestyle at a time. An increase of a few pounds in weight tends to show an increase in blood pressure. This difference can give patients a reason to focus on changes.

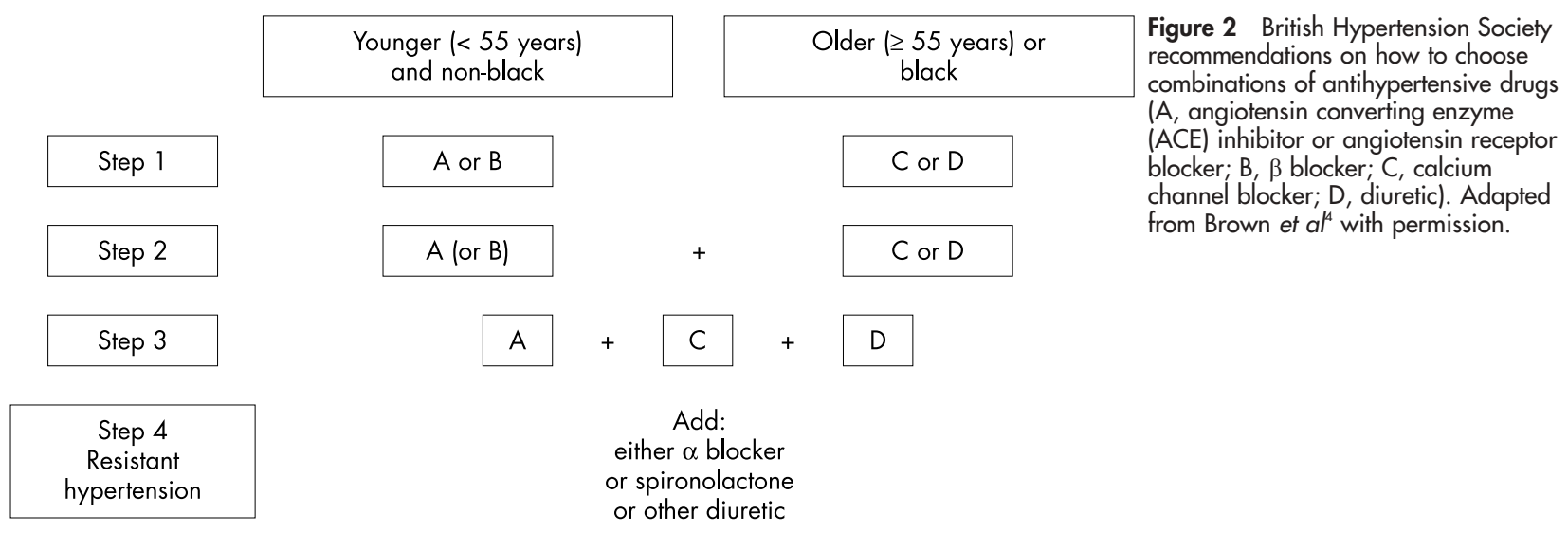


Time constraints and work pressures will inevitably mean that some of the features of this clinic cannot be translated into the general practice setting. Nevertheless, blood pressure control could be improved by using a protocol: this is probably the key aspect of the ASCOT clinic that helps us to focus our attention on lowering blood pressure. If blood pressure remains uncontrolled after three drugs are given, referral to a specialised clinic may be useful, providing that:

- Blood pressure has been taken correctly. Are all BP machines validated and calibrated? Anaeroid machines are notorious for being inaccurate. One recent study suggested that only one in 54 practices had arrangements in place for maintenance and calibration of sphygmomanometers.

- Patients are taking all drugs as prescribed. Ask the patient about side effects and whether or not they have missed any tablets, stressing that it is important for you to know this before you give them further medication. Look at concomitant medication-steroids and anti-inflammatory drugs can both cause a rise in blood pressure.

- There has been discussion of non-pharmacological measures to reduce blood pressure.

\section{CONCLUSION}

Controlling blood pressure is of paramount importance for preventing cardiovascular disease. Although treating hypertension to target level provides a challenge for health care professionals, the experience of ASCOT is that target levels can be achieved. Strict protocol adherence, reinforcement of non-pharmacological advice, and attention to patient compliance has contributed to achieving positive results in this clinic. Some of these points could be translated into the more challenging setting of primary care.

\section{REFERENCES}

1 Ramsay LE, Williams B, Johnston GD, et al. British Hypertension Society guidelines for hypertension management 1999: summary. BMJ 1999;319:630-5.

2 Primatesta $\mathbf{P}$, Brookes $M$, Poulter NR. Improved hypertension management and control: results from the health survey for England 1998. Hypertension $2001 ; 38: 827-32$

3 Sever PS, Dahlof B, Poulter NR, et al. Rationale, design, methods and baseline demography of participants of the Anglo-Scandinavian cardiac outcomes trial. ASCOT investigators. J Hypertens 2001;19:1139-47.

4 Brown MJ, Cruickshank JK, Dominiczak AF, et al. Executive Committee, British Hypertension Society. Better blood pressure control: how to combine drugs. J Hum Hypertens 2003;17:81-6.

5 Rouse A, Marshall T. The extent and implications of sphygmomanometer calibration error in primary care. J Hum Hypertens 2001;15:587-91. 